EPJ Web of Conferences 103, 02006 (2015)

DOI: 10.1051/epjconf/201510302006

(C) Owned by the authors, published by EDP Sciences, 2015

\title{
Quantum Tomography of Microwave Region
}

\author{
G.P. Miroshnichenko ${ }^{a}$ \\ ITMO University, 49 Kronverksky Pr. St. Petersburg, 197101 Russia
}

\begin{abstract}
An alternative method of reconstruction of the density matrix of microwave range created in the maser cavity is proposed. Here a technique for measuring the probability density of the quadrature field for different phases is presented. The measured quantity is the probability of difference between the numbers of atoms emitted in the excited and ground states in a sample of $\mathrm{N}$ atoms passing sequentially through the cavity. Numerical simulations performed for the numerical values of the typical parameters setting show the effectiveness of the proposed method.
\end{abstract}

Keywords: microwave region, quantum tomography, density matrix of field reconstruction, Cavity CED experiments.

Quantum properties of microsystems can be observed if weakened decoherence, destroying quantum states superposition. It is now possible ideal quantum measurement of individual quantum objects, isolated from the external environment, such as atoms, ions, photons [1, 2]. Cavity CED experiments are of particular interest for quantum information science. Here, a photons of high-Q microwave resonator act as an isolated quantum system. Methods of preparing various quantum states of photons were proposed and implemented. These are Fock states, squeezed states, "Schrodinger cat" states [3]. Quantum modes states are described in terms of quasiprobability in phase space. The task of reconstruction quasiprobability in quantum measurement is of particular importance. In the optical range there is a method of quantum tomography using optical homodyne method. In the microwave range, in Cavity CED experiments similar method is not possible due to lack of corresponding quantum detectors of microwave region. In Cavity CED experiments information about mode states we receive via the probe atom passed through the resonator. The states of emerging from the cavity probes are measured by ionization chambers. There are several methods for the extraction of quasiprobabilities from statistics of emitted from the cavity probes, in particular the Wigner function [4 - 7]. Of particular interest is a method of quantum tomography of the Wigner function, proposed in [8]. In this method, the experiments on the reconstruction of the Wigner function were carried out [911]. The method [8] shows that the Wigner function $W(\alpha)$ derived from the experiment at the point $\alpha$ in the complex plane. Wigner function associated with the difference in the probability of finding the probe in the excited and ground states by formula

$$
P_{e}-P_{g}=S p\left(D(\alpha) \rho D^{-1}(\alpha) \exp \left(i \pi a^{\dagger} a\right)\right)=\frac{W(-\alpha)}{2},
$$

\footnotetext{
${ }^{\text {a }}$ Corresponding author: gpmirosh@gmail.com
} 
where $\rho$ is the unknown matrix of density, $D(\alpha)$ is shift operator of operators $a, a^{\dagger}$ on the complex amplitude $\alpha$. This shift provides an additional coherent classical microwave field acting on the resonator containing the studying mode. For receiving parity operator $\exp \left(i \pi a^{\dagger} a\right)$ under the averaging sign used atomic Ramsey interferometer operating in the microwave range and measure the phase difference between the superposition states of the atom probe. For receiving such an operator atom probe must to interact with mode via dispersive Kerr-type effects, due to the large detuning from resonance. The phase shift at a single photon in the interferometer must be equal $\pi$. In this report, an alternative method of reconstruction of the density matrix of microwave range created in the maser cavity is proposed. Here a technique for measuring the probability density $P(x(\varphi))=\langle x(\varphi)|\rho| x(\varphi)\rangle$ of the quadrature field for different phases is presented. It is shown that this measurement is possible for a simple experimental setup that does not require finely tuned atomic interferometer and an additional source of coherent classic radiation. Input phase is performed by rotating the base after the departure of the probe atom from the cavity. The measured quantity is the probability of difference $\Delta=N_{+}-N_{-}$between the numbers of atoms emitted in the excited and ground states in a sample of $\mathrm{N}$ atoms passing sequentially through the cavity. It is shown that if the interaction time $\tau$ of the probe with a cavity is sufficiently small, then measured probability equal by the probability $P(x(\varphi))$ where the desired value $x(\varphi)$ is defined as $x(\varphi)=\frac{N_{+}-N_{-}}{2 N \tau}$. Here $N=N_{+}+N_{-}$. Numerical simulations performed for the numerical values of the parameters setting [9] show the effectiveness of the proposed method.

This work was financially supported by Ministry of Education and Science of the Russian Federation (Gosadanie No. 2014/190, Zadanie No. 1.754.2014/K).

\section{References}

1. W. Zurek, Rev. Mod. Phys. 75, P.715-775 (2003)

2. H. Haffner et al., Nature. 438, P.643-646 (2005)

3. J. M. Raimond, M. Brune, and S. Haroche, Rev. Mod. Phys. 73, P.565-582 (2001)

4. C. T. Bodendorf, G. Antesberger, M. S. Kim, and H. Walther., Phys. Rev. A. 57, P.1371-1378 (1998)

5. P. J. Bardroff, E. Mayr, W. P. Schleich, P. Domokos, M. Brune, J. M. Raimond, S. Haroche, Phys. Rev. A. 53, P. 2736-2741 (1996)

6. Martin Wilkens and Pierre Meystre, Phys. Rev. A. 43, P. 3832-3835 (1991)

7. M. Brune, S. Haroche, J. M. Raimond, L.Davidovich and N. Zagury, Phys. Rev. A. 45, P.51935214 (1992)

8. L. G. Lutterbach and L. Davidovich, Phys. Rev. Lett. 78, P.2547-2550 (1997)

9. Samuel Deleglise, Igor Dotsenko, Clement Sayrin, Julien Bernu, Michel Brune, Jean-Michel Raimond, Serge Haroche, Nature. 455, P.510-514 (2008)

10. Clement Sayrin, Igor Dotsenko, Xingxing Zhou, Bruno Peaudecerf, Theo Rybarczyk, Sebastien Gleyzes, Pierre Rouchon, Mazyar Mirrahimi, Hadis Amini, Michel Brune, Jean-Michel Raimond, Serge Haroche, Nature. 477, P.73-77 (2011)

11. T. Rybarczyk, S. Gerlich, B. Peaudecerf, M. Penasa, B. Julsgaard, K. Mølmer, S. Gleyzes, M. Brune, J.M. Raimond, S. Haroche, I. Dotsenko, arXiv:1409.0958v1 [quant-ph] 3 Sep 2014 